\title{
Deep Learning-based Finger Big ROIs Extraction for Bone Age Assessment in Smart Intelligence Systems
}

\author{
Chansu Lee and Byoung-Dai Lee \\ School of Computer Science and Engineering, Kyonggi University, Suwon, \\ 16227 Gyeoggi-Do, Korea \\ \{cslee, blee\}@kgu.ac.kr, +82-31-249-9676
}

\begin{abstract}
Tanner-Whitehouse 3 (TW3) method assesses bone age by finding 13 Regions of Interest (ROIs) from left hand X-ray image and evaluating each region. TW3 method has complicated evaluation process and many assessment factors are subjective. Hence, an automated bone age assessment system that enables objective evaluation in short period of time is required. Automatic extraction of 13 ROIs is necessary for the implementation of the automated bone age assessment system. However, direct extraction based on deep-learning mechanism can produce erroneous extraction of ROI in wrong region because many regions in left hand bone have similar appearance. To prevent this problem, finding Big ROIs from the left hand X-ray image first and extracting the corresponding ROIs from each Big ROI can increase the probability of correctly finding ROIs. This study proposes a method of finding thumb Big ROI, middle finger Big ROI and little finger Big ROI from left hand X-ray image and evaluates the performance of the method.
\end{abstract}

Key words: Bone age assessment, TW3, ROI, image processing, deep-learning, performance

\section{INTRODUCTION}

Recently, the number of children who receive therapy due to precocious puberty is increasing. Precocious puberty has diverse causes including obesity, heredity, dietary change, stress and endocrine disruptors. Precocious puberty refers to the appearance of pubertal signs that begins in early age, specifically, 8 years old in case of girls and 9 years old in case of boys. Precocious puberty not only hinders physical growth of the children but also, gives psychological distress or negatively affects the children's study as they experience puberty in too early age. In case of female children, the occurrence risk of premature menopause, breast cancer and uterine cancer increases when they become adults. However, most cases of precocious puberty either fail to recognize it or miss timing for testing. Bone age assessment is one of the diagnostic methods for the precocious puberty.

Bone age assessment analyzes the left hand X-ray image of a patient and determines his bone age. The larger the assessed bone age is than the actual age, the severer the progression of precocious puberty is. In clinical fields, bone age is assessed by Greulich-Plye (GP) method (Greulich and Pyle, 1959) and Tanner-Whitehouse 3 (TW3) method (Tanner et al., 2001). GP method has advantage that the test process is simple and it requires short amount of time. However, the standard images that become the reference of the test are distinguished in 6 months to a year interval. Hence, precise bone age evaluation is difficult. Furthermore, clinician's subjective opinion has large influence on the test outcome. On the contrary, TW3 method can more precisely determine bone age because the test can be done in 0.1 year unit, although, it requires longer test period (Griffith et al., 2007). A previous study argued that TW 3 method is more appropriate, than, GP method for children in Asian countries including Korean children because the reference of the GP method was created based on the left hand $\mathrm{X}$-ray image of western children only $(\mathrm{Oh}, 2011)$. Due the problems including complexity of assessment process, long test period and errors coming from subjective opinion, there are constant demands from the specialists in pediatrics and diagnostic radiology for automatic bone age evaluation system.

Deep-learning mechanism which is receiving increasing amount of attention in the field of intelligent information these days can be used for the implementation of the TW3 test method through the automated bone age assessment system. To implement the TW3 method as an automated system using the deep-learning mechanism, 13 bone regions should be first extracted from the left hand X-ray image. Because hand bone has many regions with similar appearance, the

Corresponding Author: Byoung-Dai Lee, School of Computer Science and Engineering, Kyonggi University, Suwon, 16227 Gyeoggi-Do, Korea, \{cslee, blee\}@kgu.ac.kr, +82-31-249-9676 
reliability of the system can largely drop with numerous wrong regions when directly extracting the 13 regions by deep-learning. In particular, 11 ROIs included in thumb, middle finger and little finger have very similar shape which makes it difficult to identify each ROI at a glance without detecting the entire hand shape. This study proposes a method of finding thumb Big ROI, middle finger Big ROI and little finger Big ROI as a preprocessing process aimed at correctly finding 11 ROIs that exist in finger region. Bone age estimation error coming from wrong region detection can be largely reduced by first finding thumb, middle finger and little finger region using the proposed method and subsequently finding ROI in each region through deep-learning.

\section{MATERIALS AND METHODS}

GP and TW3 methods: GP method and TW3 method are the two representative methods that are used for bone age test. GP method estimates bone age of a subject by comparing the left hand X-ray image of the subject with approximately 30 standard images that are marked with gender and age. GP method has an advantage that beginners without much experience can easily measure bone age and the test process is simple. However, there is a possibility that the appearance of the standard might not be appropriate for children in Asian countries because the standard images were created using the subjects exclusively composed of western children. Furthermore, the method has shortcoming that precise test results cannot be obtained because the bone age is evaluated using the standard images that are defined in 6 months to a year interval.

TW3 method obtains X-ray images of hand and wrist and analyzes the shape of 13 bones that are used in the test. The method gives each bone a maturity level according to, the predetermined standard. The method estimates bone age in 0.1 year unit based on the sum of level score that indicates maturity level of each part of the 13 bone regions. TW3 method has an advantage that the measurement outcome is more objective and accurate than GP method because the appearance of the 13 bones is compared to the bone age standard in former case. The method has shortcoming that test time is long as specialist directly analyzes the 13 bones one by one with naked eyes and large amount of time is spent for learning. An automated bone age assessment system based on TW3 method is critical to overcome this shortcoming.

Visiana company in Denmark developed BoneXpert which is an automated bone age assessment program based on the GP method (Anonymous, 2019). BoneXpert automatically extracts boundary of bones by using active
Appearance model (Cootes et al., 2002). Age of each bone is computed according to the shape score, bone density score and growth plate integrity score, based on which the bone age is modified. Although, BoneXpert finds bone regions that are necessary for bone age determination through the active appearance model, it has limitation that it cannot detect bones with shape that is very different from the previously defined model. Furthermore, it is not a technique aimed at implementing TW3 method because finding test region of TW3 method using the active appearance model is difficult.

Automated bone age assessment system, Lee et al. (2017) that was studied in Harvard University measures bone age using CNN, similar to the existing bone age estimation research. For the purpose of increasing the CNN performance, this system has a preprocessing process that captures bone only by finding mask of bone in X-ray image. Learning progresses such that bone part and no-bone part is distinguished in the X-ray image. Standardization process is executed, so that, distinction between hands and background can be easy when experiment images are entered. Mask is created by finding the parts that are perceived as bone through the previously trained model and images that exclusively capture bones are drawn. The created images are used as learning data of CNN. However, this method has lower accuracy than bone age measurement method that uses TW3 method because the former measures the bone age based on the GP method.

Finger Big ROIs extraction: For the measurement of bone age, TW3 method first finds 13 bone ROIs from the left hand X-ray image as shown in Fig. 1. In particular, 11

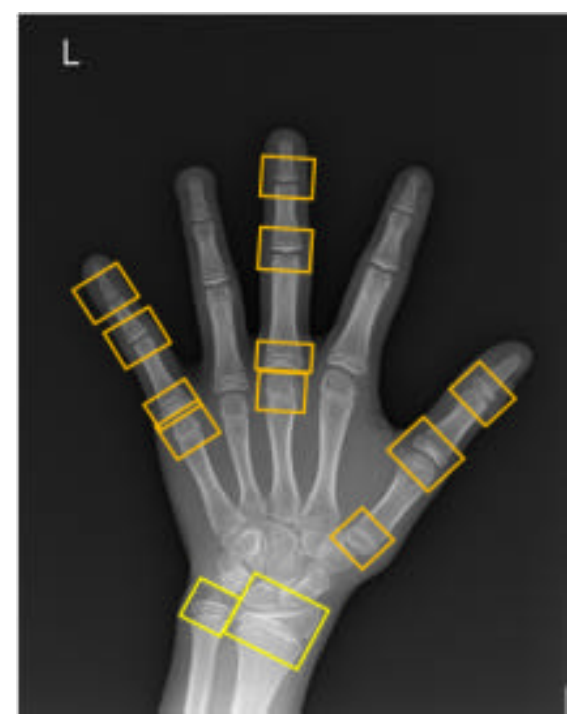

Fig. 1: The 13 ROIs for the TW3 method 
bone ROIs on thumb, middle finger and little finger have many spots that have similar appearance. As a result, direct search through deep-learning can frequently produce cases of wrong location. This study largely reduces the probability of wrongly finding 11 ROIs on fingers by finding thumb, middle finger and little finger Big ROIs first. Figure 2 shows thumb, middle finger and little finger Big ROIs.

The Big ROI extraction process is divided into two stages. In the first stage, the image is rotated, so that, the assessed bones of the Big ROI are placed vertically. To find the rotation angle of the image, the trend line of corresponding bones is found using RANSAC (Anonymous, 2018a) and the inclined angle of the trend line is calculated with an arctangent function. In the second stage, the Big ROI regions are extracted from the original X-ray image.

The finger ROIs are extracted in sequential order of middle, little and thumb fingers. To do this, we first rotate the bones of the finger region to the vertical position. In this process, an endpoint of the finger and a point representing the bone between fingers are used. To find these feature points, the convex hull method (Anonymous $2018 \mathrm{~b}$ ) is used. If the convex hull of the hand region is found by using OpenCV's Convex Hull API, a figure like the one marked in red can be obtained as shown in Fig. 3. This is the shape connecting the convex hull of the hand region.

After finding the convex hull of the hand region, convex defects can be found. Those are obtained from the outer lines of the target and the figure that connects the convex hull region. Simply put, it finds a part where the distance from the convex hull to the outer line is the

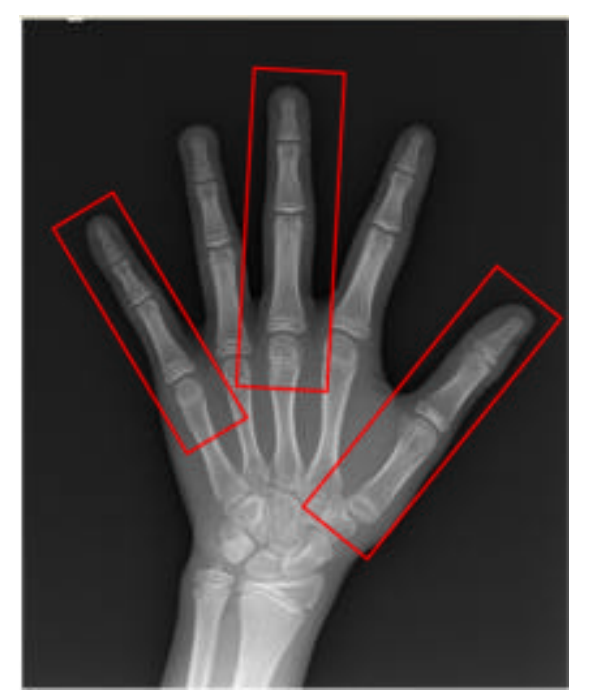

Fig. 2: Thumb, middle finger, little finger Big ROIs farthest. Figure 4 shows the feature points found through the convex defects. In Fig. 4, the orange line shows the convex defects and the yellow dots indicate the starting points, ending points and defect points of the figure which are treated as defects. The feature points can be picked this way. Thus, an endpoint of a finger and a bone point can be found. By obtaining a straight line connecting the endpoint of the finger and the bone point existing right beside it, a straight line can be drawn, as shown in Fig. 5. Then, we can roughly upright a finger using this straight line as shown in Fig. 6.

Afterwards, the image in Fig. 7 can be obtained by finding the middle points and marking them in red through the coordinates of left and right boundary points, tracing

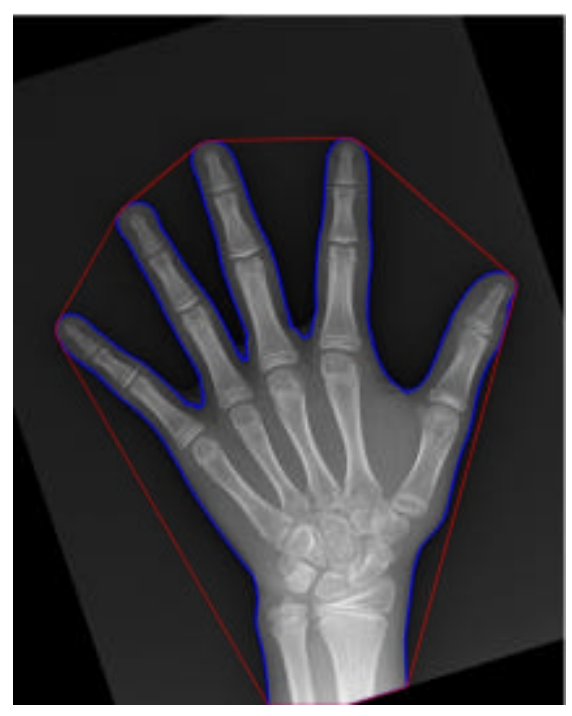

Fig. 3: Convex hull of the hand region

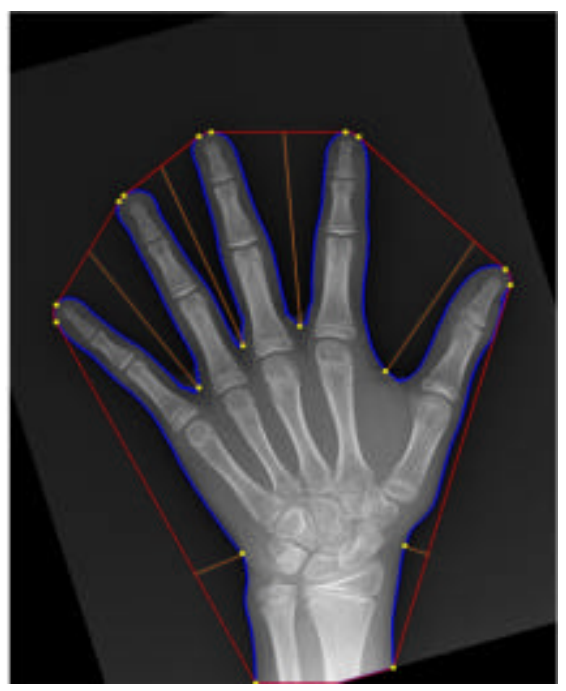

Fig. 4: Convex defects of the hand region 


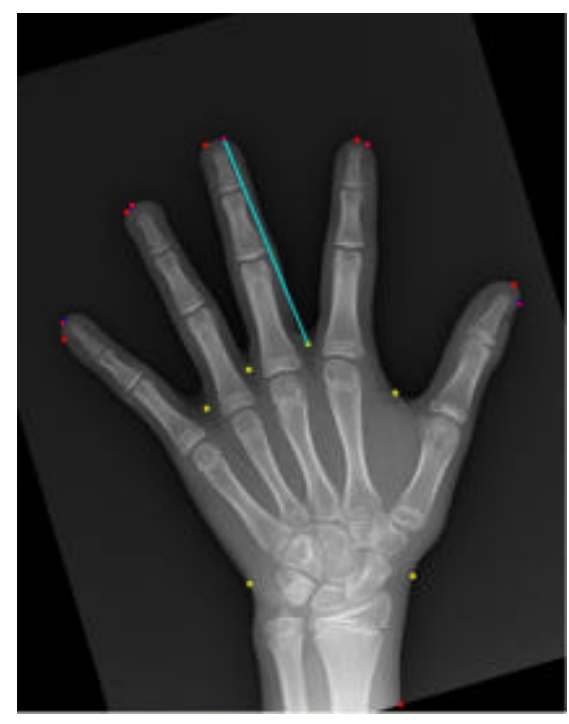

Fig. 5: A straight line connecting the endpoint of finger and the bone point

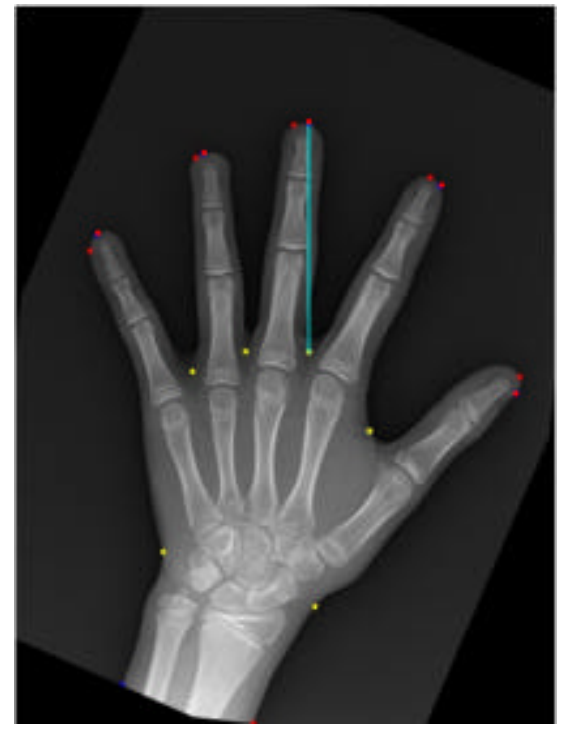

Fig. 6: Roughly upright the middle finger using the straight line

down from the endpoint of the finger along the left and right boundaries between the finger and background.

The found points are applied to RANSAC and a straight line through the middle points of the finger can be found. This straight line is a trend line showing the direction of the finger. When the image is rotated by calculating the angle to vertical, an upright image can be obtained, as shown in Fig. 8. The red straight line in the image indicates the trend line.

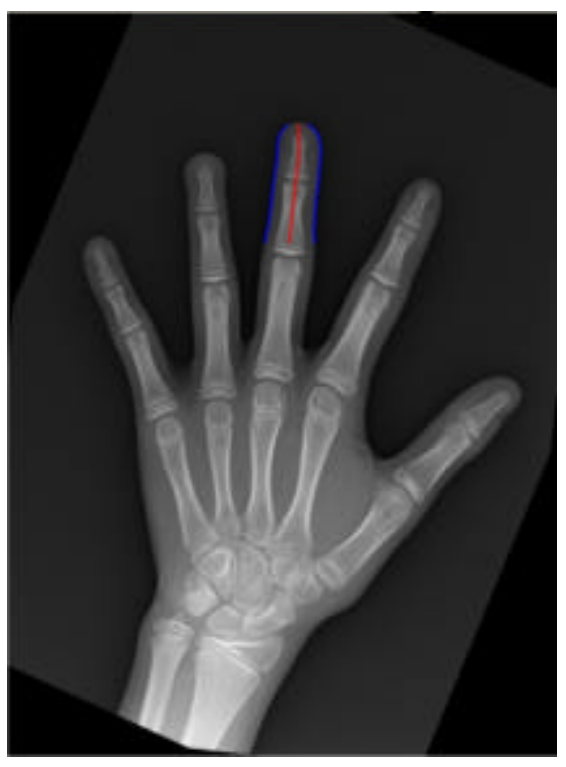

Fig. 7: Marked middle points of a finger

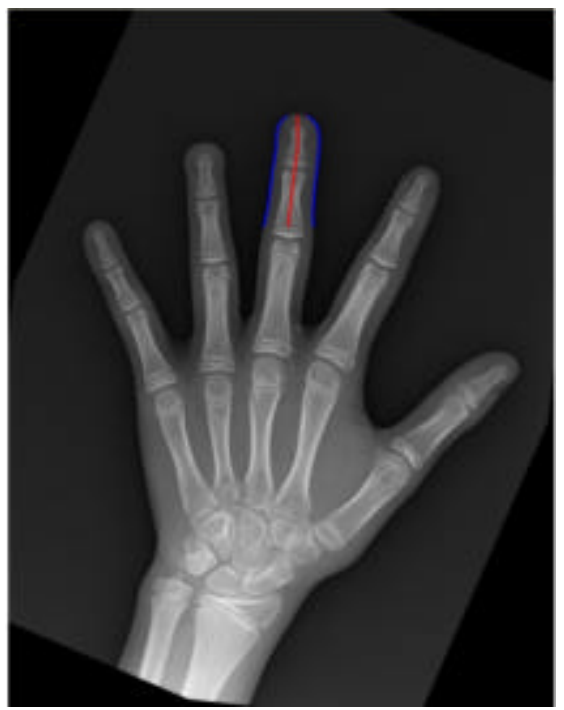

Fig. 8: Result of image rotation using trend line

Lastly, to determine the upper/lower/left/right boundaries of the Big ROI for a finger, the finger endpoint is used as the upper boundary. The bone points of both sides are used for the left and right boundaries. The center-point of the hand is used as the lower boundary. In the case of the thumb, the coordinate of highest position in the radius bone of the wrist is calculated and used as the lower boundary. Figure 9 shows the Big ROI of the middle finger and the reference points of upper/lower/left/right boundaries are marked in blue. Figure 10 displays the process of extracting the Big ROI. 


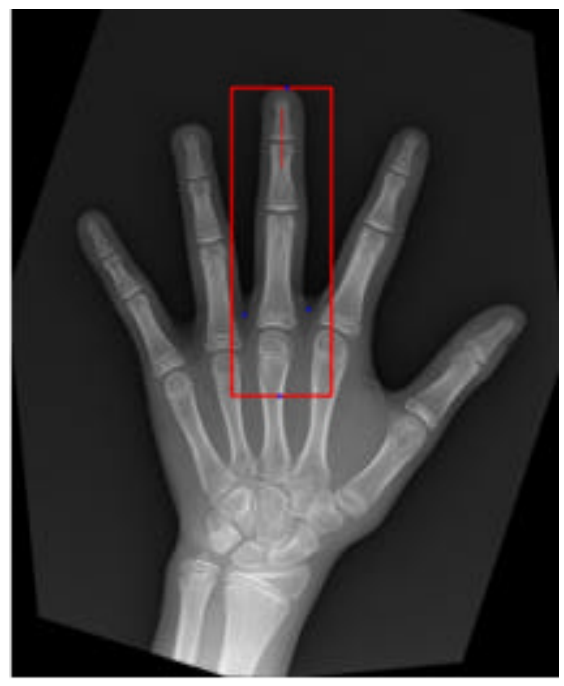

Fig. 9: Big ROI of the middle finger

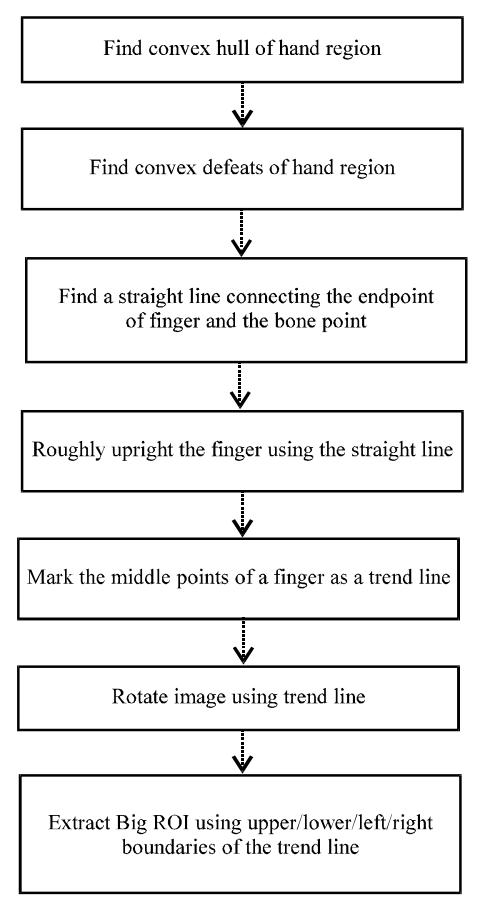

Fig. 10: Flowchart for Big ROI extraction process of a finger

\section{RESULTS AND DISCUSSION}

In this study, Big ROIs which distinguish the thumb, middle finger and little finger are extracted for bone age assessment. The process of finding trend lines of the fingers and rotating them upright has been described for extracting their respective Big ROIs. Through, this process, the trend lines of the fingers are obtained using

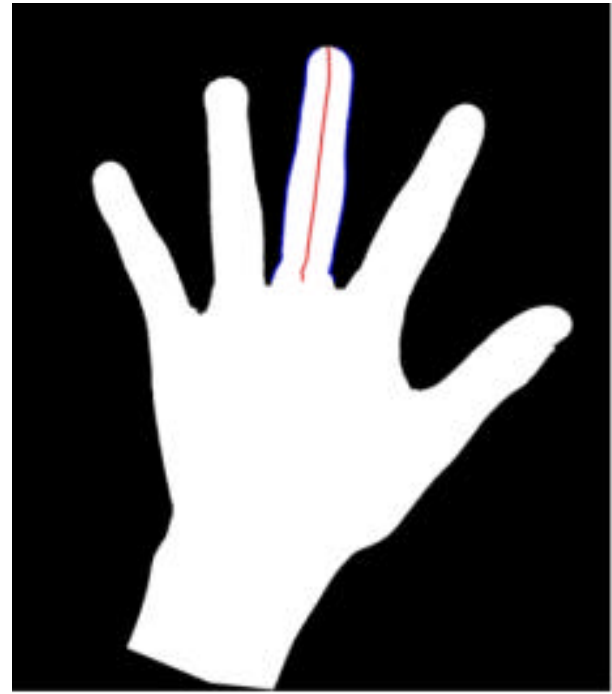

Fig. 11: Calculation of middle points of the middle finger

the RANSAC algorithm to identify rotation angles. After finding the convex hull and convex defects by using the outer lines of the hand region, the boundary of the Big $\mathrm{ROI}$ is established by finding the endpoint of the finger and the bone point between the fingers.

To find the endpoint of the finger and the bone point between fingers, the image should be binarized first. Through the binarization, we can easily obtain the boundary of the hand in the X-ray image. When binarization is performed, there may be cases where a finger becomes disconnected in the middle due to the brightness difference of the image. Here, to include the disconnected parts of the finger in the hand region, the region close to the center point of the largest region should be included.

The endpoints of the finger and the bone point between fingers are found by the convex hull method. After that, it is necessary to rotate the finger to the upright position. The method of finding the middle points is very simple. First, the boundary coordinates at both sides of the finger are identified by tracing down the outlines of both sides from the endpoint of the relevant finger. Then, the middle points are calculated from the coordinates existing at the same heights. The trend line can be found by applying RANSAC. Figure 11 shows an image marking the boundaries on both sides of finger in blue and the middle points in red.

Because the middle points have been found, the image can be rotated, so that, the finger will be placed upright, just like previous methods. Figure 12 shows images that have been rotated, so that, the thumb, middle finger and little finger are in their upright positions. 

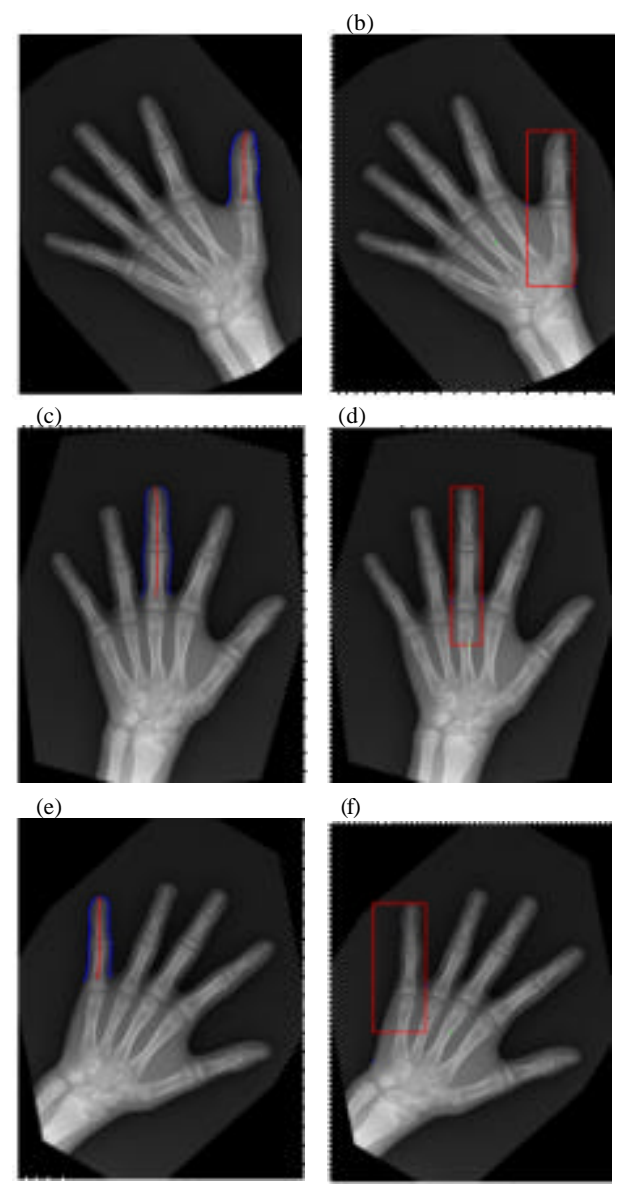

(f)

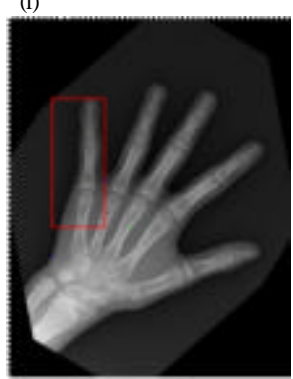

Fig. 12: a-f) Images and extracted regions of vertically erect thumb, middle finger and little finger

The endpoint of the finger, bone point between fingers and center point of the hand are the feature points used for extraction of the finger region. By using these feature points, the upper/lower/left/right boundaries of each finger region can be made. Using the middle finger as an example, the most basic method can be carried out simply by setting the endpoint of the middle finger as the upper boundary of the region, the center point as the lower boundary of the region and the points of the two bones as the left and right-side boundaries. As an exception, in the case of the thumb, if the lower boundary is set by the center point, the assessed bone can be cut off. Thus, the bone point located below the thumb is set as the lower boundary. Figure 12 shows the region boundaries established using these methods. However, in the case of extracting the finger regions by using the most basic method because the feature point positions can be found differently from the regular positions because of image peculiarities, a method extracting the region with a wider width or by stretching the upper/lower boundaries is used.

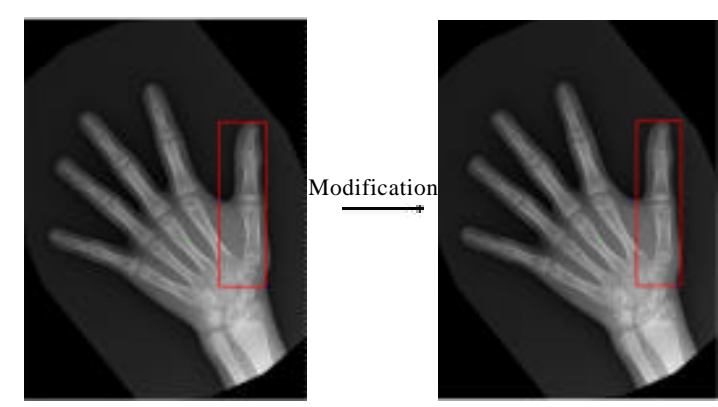

Fig. 13: Modification of the thumb Big ROI

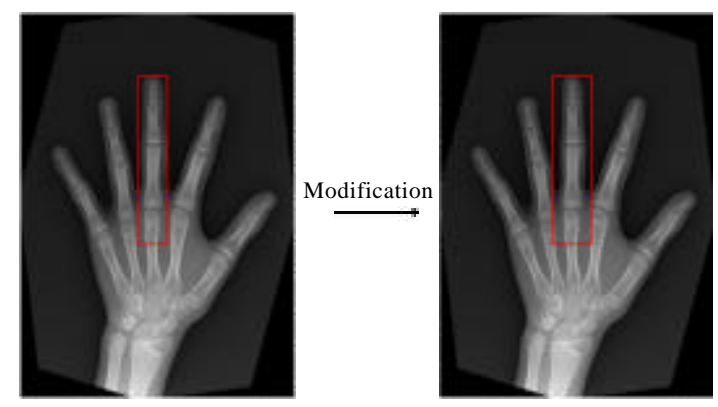

Fig. 14: Modification of the Middle finger Big ROI

In the case of the thumb, if the region is extracted with the basic method, the possibility exists that the bone point located on the right side is positioned toward the inside. Thus, a phenomenon occurs where the assessed bones are cut off. Therefore, to solve this problem, the left/right boundaries on both sides from the endpoint of the thumb is set 1.2 times the left/right boundary size of the thumb. Figure 13 shows the modification of the thumb Big ROI extraction.

In the case of middle finger region extraction, depending on the result of binarization, the points of bones on both sides can be skewed toward the inside. Therefore, the middle finger region is extracted by enlarging the basic left/right boundary width 1.3 times. Figure 14 shows the modification of the middle finger Big ROI extraction.

In the case of little finger region extraction, the point position of the left bone can be skewed noticeably to the left or the right. Furthermore because the center point can be positioned on a similar line of assessed bone, owing to the rotation, the width and height of the boundary are all transformed to supplement these problems. First, in the case of width, the difference is found between the $\mathrm{X}$-coordinates of the endpoint of the finger and the bone point located on the right side. Then, 2.5 times this value is used for the width of the left/right boundaries. In the case of upper/lower boundaries by using the Y-coordinate 

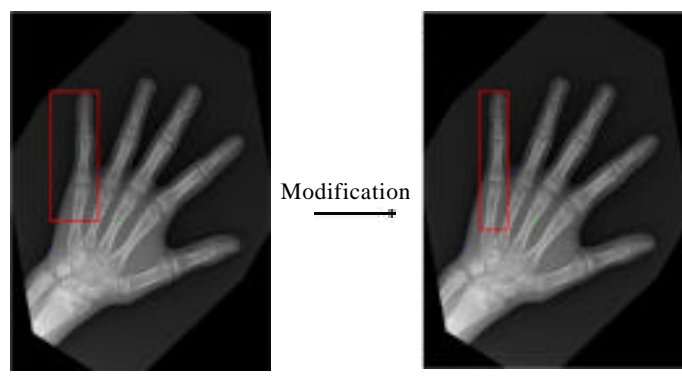

Fig. 15: Modification of the little finger Big ROI

of point of left bone and the Y-coordinate of center point, the average value of two coordinates is used as the lower boundary. Figure 15 shows the modification of the little finger Big ROI extraction.

\section{CONCLUSION}

This study examined a method of extracting thumb Big ROI, middle finger Big ROI and little finger Big ROI by using TW3 method as a preprocessing method of measuring bone age from left hand X-ray image. The TW3 method measures the bone age of a subject by finding 13 ROIs in the left hand X-ray image and obtaining the average of the measured maturity of each ROI. For automatization of this measurement process, 13 regions should be first extracted from the left hand X-ray image. Due to the characteristics of hand bones that have many regions with similar appearance, the probability of judging wrong region as a certain ROI becomes high when directly estimating 13 ROI locations through deep-learning. To prevent this problem, thumb Big ROI, middle finger Big ROI and little finger Big ROI should be first found in the X-ray image and then ROIs that are contained in each Big ROI should be found. This study proposed a method of searching for Big ROIs and evaluated the performance. Development of an automated bone age assessment system through deep-learning by using the ROIs that were extracted from these Big ROIs remains as a future work.

\section{ACKNOWLEDGEMENTS}

This research was supported by Kyonggi University Research Grant 2018.

\section{REFERENCES}

Anonymous, 2018a. Graham scan. Wikimedia Foundation, Inc., San Francisco, California, USA. https://en. wikipedia.org/wiki/Graham_scan

Anonymous, 2018b. Random sample consensus. Wikimedia Foundation Inc., San Francisco, California, USA.https://en.wikipedia. org/wiki/ Random_sample_consensus

Anonymous, 2019. Precise bone age-for better patient care. Visiana, Agern Alle, Horsholm, Denmark. https://www.bonexpert.com/

Cootes, T.F., G.V. Wheeler, K.N. Walker and C.J. Taylor, 2002. View-based active appearance models. Image Vision Comput., 20: 657-664.

Greulich, W.W. and S.I. Pyle, 1959. Radiograph Atlas of Skeletal Development of the Hand and Wrist. 2nd Edn., Stanford University Press, Palo Alto, California, USA., ISBN:9780804703987, Pages: 272.

Griffith, J.F., J.C.Y. Cheng and E. Wong, 2007. Are western skeletal age standards applicable to the Hong Kong Chinese population? A comparison of the Greulich and Pyle method and the Tanner and Whitehouse method. Hong Kong Med. J., 13: S28-S32.

Lee, H., S. Tajmir, J. Lee, M. Zissen and B.A. Yeshiwas et al., 2017. Fully automated deep learning system for bone age assessment. J. Digital Imaging, 30: 427-441.

Oh, Y.J., 2011. Development and evaluation of semiautomatic bone age estimation method. Master Thesis, Ewha Womans University, Seoul, South Korea.

Tanner, J.M., M.J.R. Healy, N. Cameron and H. Goldstein, 2001. Assessment of Skeletal Maturity and Prediction of Adult Height (TW3 Method). 3rd Edn., W.B. Saunders Company, Philadelphia, ISBN: 9780702025112, Pages: 110. 